\title{
Madurez psicológica y disciplina en el logro de metas. Relato de vida de María Torcates
}

\author{
Psychological maturity and discipline in the achievement of goals. \\ Life story of María Torcates \\ Maturidade psicológica e disciplina no alcance de metas. \\ História de vida de María Torcates
}

\author{
María Torcates \\ marisatorcates@gmail.com
}

Universidad de Carabobo, Valencia, Venezuela

\section{Mirian Rosales}

mirianros.95@hotmail.com

Universidad de Carabobo, Valencia, Venezuela
RESUMEN

A pesar de todas las condiciones adversas que le pueden ocurrir a un estudiante universitario, éste persiste en su meta. La intencionalidad de este estudio fue comprender su madurez psicológica y disciplina en el logro de las metas. Su metodología fue cualitativa fenomenológica con relato de vida del enfoque biográfico de Legrand (1993). De la fase de análisis se obtuvieron dos grandes bloques de sentido respectivamente a modo gestálgico, se produjeron dos aéreas significativas e importantes en el estudiante universitario uno su madurez psicológica, y otro, su disciplina en el logro de sus metas. Pone al frente a una narrativa de vida que hace ver que, en lo cotidiano, hay cosas que se deben investigar porque pasan desapercibidas; con este trabajo se percibió que más allá de llegar al aula de clases, rendir, pasar los semestres, egresar, se esconden elementos puntuales como son la madurez psicológica y la disciplina.

Palabras clave:

Madurez psicológica; disciplina; estudiante universitario; relato de vida; logro de las metas
ABSTRACT

Despite all the adverse conditions that can happen to a university student he persists in his goal. The intention of this study was to understand their psychological maturity and discipline in achieving goals. His methodology was qualitative phenomenological with a life story from the biographical approach of Legrand (1993). From the analysis phase, two large blocks of meaning were obtained respectively in a gestural way, there were two significant and important areas in the university student, one his psychological maturity, and the other, his discipline in the achievement of his goals. It puts in front of a life narrative that shows that, in everyday life, there are things that must be investigated because they go unnoticed; With this work it was perceived that beyond reaching the classroom, performing, spending the semesters, graduating, there are specific elements such as psychological maturity and discipline.

Key words:

Psychological maturity; discipline; university student; life story; goal achievement
RESUMO

Apesar de todas as condições adversas que podem acontecer a um estudante universitário, ele persiste em seu objetivo. A intenção deste estudo foi compreender sua maturidade psicológica e disciplina no alcance de metas. Sua metodologia foi qualitativa fenomenológica com uma história de vida a partir da abordagem biográfica de Legrand (1993). A partir da fase de análise, foram obtidos dois grandes blocos de sentido respectivamente de forma gestual, duas áreas significativas e importantes foram produzidas no universitário, uma sua maturidade psicológica, e a outra, sua disciplina no alcance de seus objetivos. Colocase diante de uma narrativa de vida que mostra que, no cotidiano, há coisas que devem ser investigadas porque passam despercebidas; Com esse trabalho percebeu-se que além de chegar à sala de aula, atuar, passar os semestres, se formar, existem elementos específicos como maturidade psicológica e disciplina.

Palavras-chave:

Maturidade psicológica; disciplina; estudante universitário; história de vida; cumprimento de metas 


\section{INTRODUCCIÓN}

Venezuela, en la actualidad, es un país de grandes retos y frente a esta realidad que se genera en medio de una crisis social, política y económica en todos los niveles; el sector universitario ha sido afectado, al punto que en las universidades públicas los estudiantes parecieran que ya no representan su razón de ser, frase que siempre está en el discurso de unos y otros.

El estudiante universitario aún en medio de tantas dificultades, sigue adelante cuando su prioridad está dirigida a culminar la formación académica de pregrado hasta egresar como profesional del área de su preferencia.

Avanzar en ese propósito es difícil en estos tiempos, por tal motivo es esencial mantener un orden de vida, y tener presente que ser constante en la disciplina individual siempre aliviará este transitar.

Al efecto, es importante señalar lo que afirman Pérez y Merino (publicado 2008), (actualizado 2012), la disciplina es el método, la guía o el saber de una persona, sobre todo en lo referente a cuestiones morales. El concepto también se utiliza para referirse a una rama científica o artística.

A su vez, Aguilar (2012), quien refuerza esa definición y destaca la importancia de la disciplina personal cuando expresa que, un ejército debe actuar con disciplina, y en los deportes de equipo no se consiguen resultados sino a base de disciplina, táctica y entrenamiento.

Es válido señalar que desde tiempos antiguos la disciplina ha sido tema de controversia por las implicaciones que encierra este término para hombres y mujeres en su transitar de vida.

A su vez, con los aprendizajes que el ser humano ha obtenido, como lo expresa Vethencourt (2009),

va conformando una serie de principios para su actuación personal en distintos campos, vale decir que en todas las etapas de su vida como ser humano el hombre evoluciona y de esta manera alcanza su propio nivel de crecimiento espiritual, moral y ético todo lo cual con el devenir de los años va encausando su madurez hasta segmentar buenas decisiones para alcanzar sus logros (...) (p.2).

Por lo tanto, es preciso reiterar que ser disciplinados en cada momento del accionar de vida, es altamente significativo porque de esta manera se forjan los sueños, plasman ideas y diseñan proyectos que encaminan al éxito y a obtener resultados fructíferos, fundamentados en la disciplina como guía.

De este modo, la investigación tiene como propósito, comprender al estudiante universitario, su madurez psicológica y la disciplina en el logro de las metas desde el relato de vida de María Torcates.

Con el desarrollo de este estudio se pretende, generar herramientas bajo el enfoque social, las cuales fomentará un acercamiento al entorno que circunda a escuelas y otras instituciones educativas, a los fines de hacer posible el mejoramiento de la relación de los padres y sus representados, de esa forma demostrar la disposición que los orientadores deben manejar como contribución en la práctica diaria para formar mejores ciudadanos, críticos y de igual modo con un pensamiento abierto que más allá del interés personal coadyuve a forjar un mejor país donde todos apostemos al rescate de los valores y al reencuentro.

\section{Marco teórico}

\section{La enseñanza y su circunstancia}

Para Aguilar (2012), la enseñanza y su circunstancia, indica que sin silencio, compostura, atención y estricto orden en clase es imposible aprender. La disciplina personal y escolar debe estar presente desde temprana edad, delineando un orden para la vida futura como valor fundamental para la persona.

Como seres pensantes se crean necesidades básicas para subsistir, más allá del alimento y la 
vestimenta, existen otras prioridades que hacen posible vivir en sociedad y todos deben ajustarse a los principios, que en existencia son importantes en la medida que son fundamentales para un mejor convivir y desempeñar en el rol que corresponde según sea el caso.

Todos esos elementos conforman un complejo tejido que se forma de la relación entre valores personales, familiares y socio-culturales. Cuando se exagera, los valores materiales entran en contradicción con los espirituales y los principios morales.

Así surgen las contradicciones entre los individuos. Las mismas vienen dadas cuando el comportamiento personal no guarda coincidencias con las actitudes y conductas que una determinada sociedad considera indispensables para la convivencia, el orden y el bien general.

\section{Educar para la ciudadanía}

Camps (2007) define la ciudadanía como el reconocimiento y la comprensión de unos derechos fundamentales (libertad e igualdad) que constituyen elementos básicos para desarrollar un particular proyecto de vida (p. 19).

Por lo tanto, la ciudadanía es el reconocimiento de las obligaciones y responsabilidades que vinculan con el denominado interés común. Ser un buen ciudadano significa asumir como sujetos tanto de derechos, como de deberes. Sujetos autónomos cuya autonomía, más que ser la pretensión individualista de hacer lo que se quiere, es asumir la libertad individual en relación con la construcción y mantenimiento del bien colectivo.

En este sentido, Camps (2007) dice que, "falta un proceso establecido y reconocido destinado a inculcar hábitos, a formar el carácter, a crear un ethos común y compartido en el que los individuos reconozcan y alimenten su identidad moral". (p.19).

Si se entiende la ciudadanía como una disposición para lo común, Camps (2007) resalta que, es evidente que tal disposición no se desarrolla espontáneamente, antes bien, resulta necesario formarla y constituirla en el carácter del ser humano.

\section{Madurez psicológica}

Vethencourt (2009) señala que una persona alcanza la madurez psicológica no porque sea adulto, ya que, esa condición es resultado de todo un proceso que vive cada individuo desde su etapa inicial, es decir desde el nacimiento y cuando sabe salir de su propia realidad, para entender la realidad de otra persona, de esta forma se evidencia, el equilibrio en su salud mental.

A su vez, Vethencourt (2009) enfatiza que la salud mental de alguna manera tiene que ver con un tipo de relación cierta entre el individuo y el mundo y del individuo consigo mismo.

Así mismo, desde el psicoanálisis y en el marco de su propia experiencia, refiere Vethencourt (2009), que algunos puntos del mundo interior, se expresan en un descubrimiento del psicoanálisis, el cual trata de la lenta y riesgosa construcción de la subjetividad.

Es preciso señalar que Vethencourt (2009), desde el psicoanálisis enfatiza en; la importancia de todos los procesos que van compactando la estructura de la personalidad, por ejemplo, el sujeto de manera aceptable comprende la realidad, de manera especial su propia realidad psicológica, su autoestima y su relación personal.

La plenitud y el estado mental saludable, son factores fundamentales para el buen desempeño de la persona; es decir tener una madurez psicológica desde el "yo central", en consideración a que como orientador se debe desarrollar un proceso de ayuda y de atención ética, el cual debe ver y escuchar más allá de las limitaciones, carencias o contingencias que pueda tener el Hombre.

\section{MÉTODO}

La investigación estuvo enmarcada dentro del paradigma cualitativo fenomenológico, con un método relato de vida y un enfoque biográfico.

Adicional, el diseño que se utilizó para el desarrollo de la investigación estuvo basada en las fases del relato de vida, el cual estuvo conformada por tres fases; fase 1 , fase de contactos, negociaciones y contratos; fase 2 , fase 
de consentimiento de los participantes y fase 3 , fase de lógica y método de análisis del relato de vida.

La fiabilidad del relato de vida, que se usó en el estudio, fueron las planteadas por Catani (1982), el primer criterio usado para la validación fueron las referencias de la vida cotidiana, el segundo criterio fueron los encuentros constantes con el narrador y el tercer criterio, la observación.

Ese planteamiento teórico, aplica para el relato de vida de la narradora, cuando en su cotidianidad refleja un orden que está presente en cada episodio de su propia historia, desde la infancia más temprana hasta este tiempo en su contexto laboral educativo. Es decir, que en su transitar de vida, va aprendiendo en cada etapa a diferenciar la subjetividad, a partir de su conducta tanto en el hogar, la academia y su espacio de trabajo, lo que muestra todo un proceso inherente al caso personal que así va moldeando su madurez psicológica.

\section{RESULTADOS}

\section{Relato de vida de María Torcates}

Hoy salí de mi casa para la universidad a las 6:00 de la mañana caminé hasta la parada ubicada en la Avenida Universidad frente a El Carabobeño, no esperé mucho tiempo porque la camioneta llegó rápido y en el arco estaba a las 6:40 de la mañana, pero hubo una demora por deficiencia en el transporte universitario, pero inmediatamente llegaron 4 unidades seguidas y el acceso se hizo de manera ordenada. Una hora después de haber salido de mi casa llegué a la facultad, estuve un rato esperando a la profesora Marisela Moreno de Práctica II quien al llegar dio explicaciones sobre la materia, luego llegó la profesora Vivian González de Seminario quien también dictó su clase.
María Torcaste, reseño el orden de lo que es su agenda en su rutina de cada día lo que demostró su disciplina personal; De esa manera se evidenció que hay capacidad para enfrentarse al mundo actual pleno de dificultades, entre ellas se tienen; el transporte, inseguridad y la falta de dinero en efectivo; siendo esto, solamente parte de las exigencias que se le presentan al estudiante universitario en el día a día cuando su meta es culminar una carrera universitaria. Por lo tanto, María, no se detuvo, aunque hayan existido problemas en el trasporte porque para ella superar vicisitudes es una condición innata.

De esa manera por el orden personal de sus actividades, María, logró los objetivos propuestos, venciendo todas las dificultades que se le presentan a diario por la situación del país, ya que, con disciplina se puede alcanzar la meta que cada individuo se propone en estos tiempos de crisis, todos los venezolanos y en particular la juventud estudiosa debe poner un poco más de empeño y acostumbrarse a hilvanar sus ideas para salir airoso de estas exigencias.

En este sentido, en cuanto a lo que se refiere a la madurez psicológica tal como lo plantea Vethencourt (2009), cuando el autor expresa que; "la dirección de la salud mental, avanza desde la valoración de lo puramente necesario hacia la necesidad de lo valioso" (s.p).

Continuando con el relato de vida de María, reseñó:

Terminando el horario en FACEUC regresé a mi casa y posterior al almuerzo salí a dictar mis clases en la academia donde laboro. A primera hora con el grupo de mis niñas pequeñas (3 a 5 años) quienes mantienen un buen comportamiento además estaban súper felices porque hicimos un juego para identificar los puntos del salón atendí al 2 grupo de niñas adolescentes de (10 a 12 años) no fue mi mejor tarde, la indisciplina que tienen estas niñas, no atienden a clase y hacen bullying a su compañera con Síndrome de Down. Al 
llamado de atención no atendían y para retomar el orden apliqué 5 minutos de preparación física sin embargo recriminé la mala actitud que continuaron en el salón de baile advirtiéndoles que nuevamente hablaría con sus padres por no atender las recomendaciones que se les hicieron en una reunión efectuada la semana pasada para que esta situación no se repita.

Cabe destacar que, en la actualidad en Venezuela, el estudiante universitario tiene que trabajar y estudiar, lo cual es una manera responsable de cubrir sus propios gastos, al tiempo de atender a un compromiso de vida para alcanzar un mejor futuro. Tal como lo señala Camps (2007), la ciudadanía es el reconocimiento de unas obligaciones y responsabilidades que nos vinculan con este denominado interés común" (p. 18).

Seguidamente, María expresó un incidente que tuvo con un grupo de alumnas; el cual ocurrió con su segundo grupo, ellas son niñas adolescentes que provocan un contra tiempo a causa de su comportamiento de indisciplina lo que representó incomodidad. "Canalicé esa situación aplicando 5 minutos adicionales de preparación física haciéndoles ver que es una forma de sancionar su conducta". Se evidenció, en este caso que María, tiene un perfil definido acerca de la disciplina, el orden y el respeto que cada sujeto debe mantener hacia las demás personas, así mismo, mostró la actitud que aplica en su condición como docente, que, con el llamado de atención y la sanción de extender los ejercicios físicos sin suspender la clase pautada para ese día, cumplió con advertir a las alumnas la falta que cometieron contra una de sus compañeras.

Aunado a ello, María no fue suficientemente severa quizás por su propia convicción, aun cuando como autoridad del aula más que la amonestación pudo ordenar la salida temporal de las jóvenes indisciplinadas, lo que viene siendo una sanción moral a su mal comportamiento. Con la debida advertencia recriminó su actitud y quedo pendiente una conversación con los padres quienes ya habían sido advertidos del mal comportamiento de sus representadas.

Adicional, se apreció cómo van surgiendo otros temas que de acuerdo a los enfoques teóricos. Se trata de aspectos relevantes como la importancia de cada uno de ellos, que se refieren a la disciplina personal como forma de vida, a la disciplina en el aula que es factor fundamental para el aprendizaje y la madurez psicológica que manifestó María en su relato de vida. 


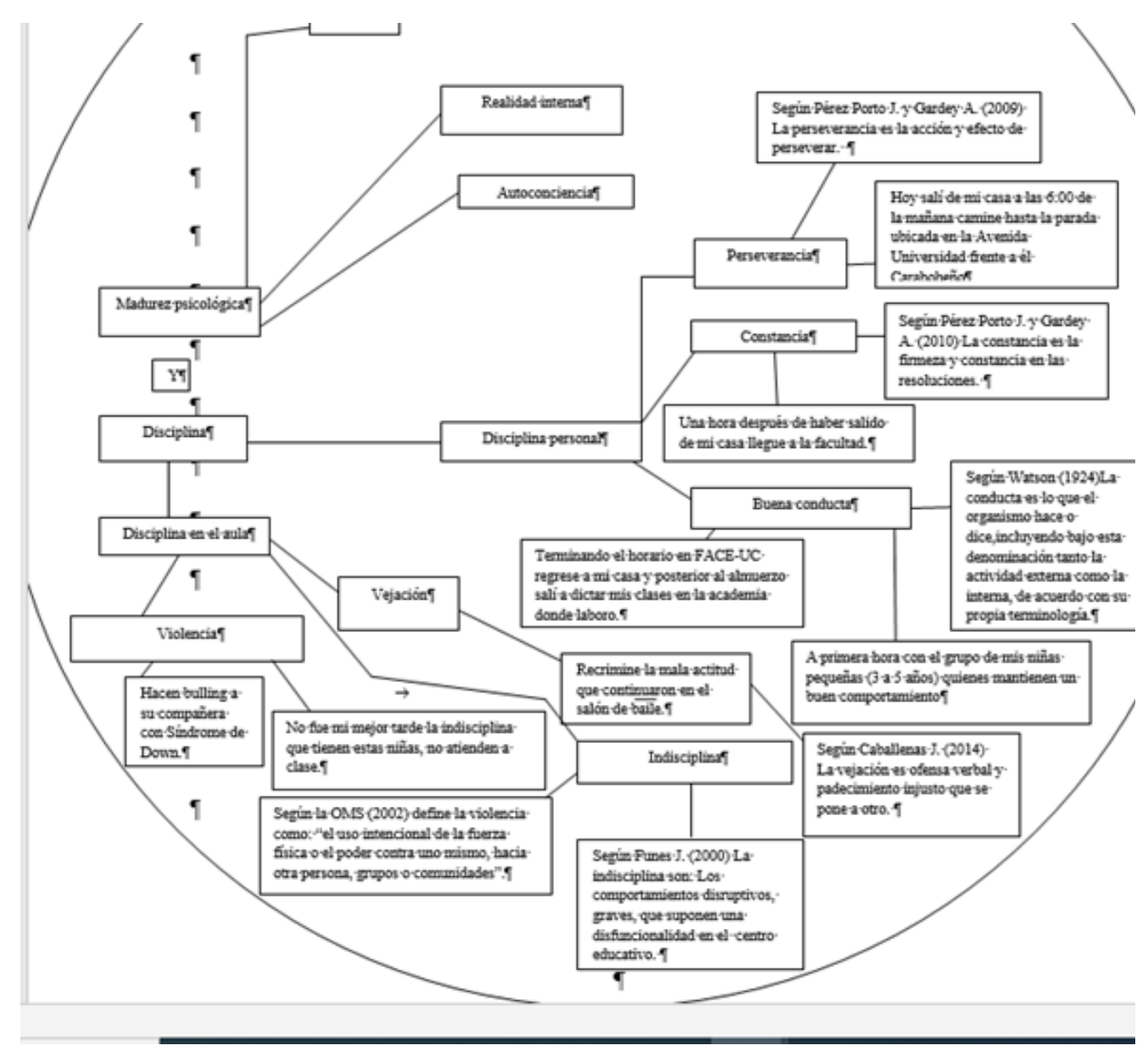

Figura 1. Enfoques teóricos. Madurez psicológica, disciplina personal y en el aula.

\section{CONCLUSIONES}

El fundamento principal de la investigación realizada sobre la disciplina (de importante significado en la vida personal del individuo), complemento el conocimiento sobre el comportamiento del Hombre, como punto central de su mundo interno y también como parte de la compleja realidad que ubica más allá de uno mismos.

La investigación, tuvo mucha importancia para la orientación, porque con el estudio realizado se llevó a conocer en profundidad el valor que representan la disciplina, la autodisciplina, el comportamiento ético y la madurez psicológica en la existencia de cada individuo.
El conocimiento aprendido a partir de la revisión bibliográfica de reconocidos teóricos en cada etapa de la historia y desde ámbitos diversos, confirmó que este estudio vincula directamente con los distintos procesos biopsicosociales que desde su nacimiento y en su transcurso de vida, van consolidando la madurez psicológica que permitirán al individuo colocarse más allá de su propia realidad y comprender la realidad del otro. Esto sentará las bases para la acertada toma de decisiones, en cualquier momento importante de su existencia para cada ser humano. El mejor ejemplo: los estudiantes universitarios que hacen frente a las vicisitudes de su día a día para seguir formándose y egresar como profesionales. 
Para finalizar, de las vivencias contenidas en la investigación, la situación actual de Venezuela ayuda a formar criterios definidos y críticas constructivas ante las políticas públicas inconvenientes para la sociedad contemporánea.

\section{REFERENCIAS}

Aguilar, J. (2012). Artículo La enseñanza y su circunstancia. [Página web]. Recuperado de https://www.libertaddigital.com/opinion/ ideas/la-convivencia-antes-disciplina, Málaga, España (13-03-2012, sin paginación) periódico digital descargado el 10/07/2018

Camps, V (2007, p. 85) Educar para la ciudadanía. Colección Foro Educativo, N. ${ }^{\circ} 3$, de la Ed. Fundación ECOEM Barcelona, España. Descargado: 26/06/2018
Catani, M. y Mazé S. (1982). Tante Suzanne: une histoire de vie sociale. París: Librairie des Méridiens

Legrand M. (1993) Relatos de Vida en Enfoque biográfico. Paris: Hombres en Perspectivas. (Apuntes V. González, p.7) Descargado el $14 / 07 / 2018$

Pérez, J., y Merino, J. (publicado 2008), (actualizado 2012). Disciplina (Página web) Recuperado de (https://definicion.de/disciplina/), en Blogspot Glorario de Definiciones, (sf, sin paginación) Descargado: 25/06/2018

Vethencourt, J. (2009,). La madurez psicológica. Caracas, Venezuela. Editorial: Heterotopias edición especial Descargado el 20/06/20018) 


\section{Currículo de Autores}

\section{Ángel Martínez}

Licenciado en Educación, mención Orientación Universidad de Carabobo (2019). Profesor de Inglés Instituto Loscher (2017).

\section{Betania González}

Licenciada en Psicología. Especialista en Psicoeducación. Experiencia en atención prehospitalaria y evaluación psicológica en niños desde preescolar hasta primaria. Certificación en aplicación de test organizacionales (Wartegg).

\section{Eliza Escobar}

Licenciada en Psicología. Facilitadora de proyectos para grupos de rescate fomentando la motivación y el autoestima, certificada en evaluación pre-empleo y test de Wartegg, en habilidades terapéuticas de Carl Rogers.

\section{Elsa De La Torre}

Licenciada en Educación, mención Orientación Universidad de Carabobo. Diplomado básico DINA (Desarrollo Integral para Niños, Niñas y Adolescentes) y RENACENIV (Red Nacional Cristiana Al Servicio de Niños niñas y Adolescente venezolano) Iglesia Bautista - Valencia (2013).

\section{Estephanny Guaimara}

Licenciada en Educación,mención Orientación Universidad de Carabobo. Profesora de Primaria en Unidad Educativa Virgen María, Guacara, Carabobo.

\section{María Torcates}

Licenciada en Educación, mención Orientación, por la Universidad de Carabobo, Valencia, Venezuela. 


\section{Currículo de Autores}

\section{Mirian Rosales}

Licenciada en Educación, Mención Orientación, por la Universidad de Carabobo.

\section{Simonne Gennaro}

Psicólogo. Ha realizado: ·Diplomado básico DINA (Desarrollo Integral para Niños, Niñas y Adolescentes). RENACENIV (Red Nacional Cristiana al Servicio de Niños niñas y Adolescente venezolano) Iglesia Bautista - Valencia (2013). Crece y Aprende (Formación para la niñez) por Asociaciones Bíblicas, Calabozo, Estado Guárico (2012). Un trato por un buen trato (prevención de violencia hacia la niñez) Calabozo, Estado Guárico (2014). RENACENIV y Unicef. 